\title{
Coupling-Matrix Design of Dual/Triple-Band Uni-Planar Filters
}

\author{
Marjan Mokhtaari ${ }^{1}$, Jens Bornemann ${ }^{1}$ and Smain Amari ${ }^{2}$ \\ ${ }^{1}$ University of Victoria, Victoria, BC, Canada V8W 3P6 \\ ${ }^{2}$ Royal Military College of Canada, Kingston, ON, Canada K7K 7B4
}

\begin{abstract}
Dual-band and triple-band filters in uni-planar technology are designed using cross-coupled networks represented by coupling matrices. The multiband response is created by placing transmission zeros within the bandwidth of a wideband filter. Realizable planar topologies can be imposed and associated coupling coefficients enforced during optimization. The design method is demonstrated using three different planar resonator configurations: hairpin, open-loop and quasi-dualmode resonators. Designs are verified by commercially available software packages and, in principle, by measurements.
\end{abstract}

Index Terms - Filter design, coupling matrix, dual-band filters, microstrip filters, planar resonators.

\section{INTRODUCTION}

Dual-band and multi-band filters are in demand for multifrequency applications in wireless communication, e.g. [1]. In planar circuitry, they can be implemented using four basic approaches: First, by switching between two separate filters at two different frequencies [1]; this approach increases size and cost. Secondly, by employing stubs to create transmission zeros, which separate passbands [2]; as this is essentially a stopband approach, far-out-of-band rejection is impossible to achieve. Thirdly, by using stepped impedance resonators, e.g. [3]; however, it is often difficult to achieve proper coupling coefficients for a simultaneous, yet independent control of both midband frequencies and bandwidths. The fourth approach consists of coupled resonator pairs [4] but suffers from an independent option for the locations of transmission zeros.

Therefore, in this paper, we propose cross-coupled networks, which are characterized by a coupling matrix similar to those used in waveguide topologies, e.g. [5], [6], for the design of uni-planar dual-band and triple-band filters. In order to demonstrate that the basic design is independent of the type of planar resonator, three different commonly used planar resonator configurations are presented as examples: hairpin resonators [7], open-loop resonators [8] and quasidual-mode resonators [9].

\section{DESIGN}

For given passband frequencies and associated bandwidths, the design procedure commences by initially assuming that a wideband filter covering all passbands will be created. The underlying approach to create a coupling matrix is presented in [6], [10] and will not be repeated here. Its main advantages with respect to the design of multiband filters are, first, that the topology of coupled resonators can be specified in advance and, secondly, that the signs and limits of coupling coefficients can be enforced during optimization.

Since the maximum number $N$ of realizable transmission zeros is dictated by the topology, any number $n \leq N$ of transmission zeros can now be placed within the initial broad passband in order to separate individual passbands. Optimization [10] is employed to adjust the entries of the coupling matrix. Their maxima and minima can be controlled via closed-form expressions for the electric and magnetic coupling coefficients of, e.g., open-loop resonators [11].

As a design example, let us consider a four-pole crosscoupled dual-band filter utilizing hairpin resonators on RT6006 substrate. The center frequency of the wideband approach is $3 \mathrm{GHz}$, and 3.5 percent bandwidth in each passband is to be retained after placing transmission zeros at 2.95 $\mathrm{GHz}$ and $3.05 \mathrm{GHz}$. Following the above procedure and specifying the topology such that the two transmission zeros be created by cross-coupling between the first and the last (fourth) resonators, the coupling matrix for this filter is found as:

$$
\mathrm{M}=\left[\begin{array}{cccccc}
0 & 0.6033 & 0 & 0 & 0 & 0 \\
0.6033 & 0 & 0.8998 & 0 & 0.4040 & 0 \\
0 & 0.8998 & 0 & -0.0063 & 0 & 0 \\
0 & 0 & -0.0063 & 0 & 0.8998 & 0 \\
0 & 0.4040 & 0 & 0.8998 & 0 & 0.6033 \\
0 & 0 & 0 & 0 & 0.6033 & 0
\end{array}\right]
$$

Fig. 1 shows the simulated performance of this filter obtained from the coupling matrix in (1).

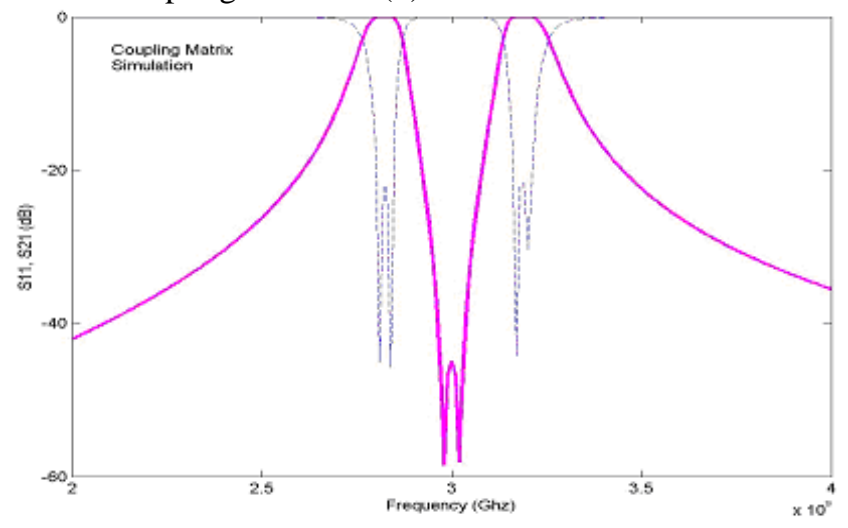

Fig. 1. Response of dual-band filter from (1).

A possible realization is shown in the inset of Fig. 2. The response in Fig. 2 is obtained by equating the coupling coefficients of (1) with those in the actual structure. Of 
course, the achievable precision is limited in this step and slight differences between responses of the coupling matrix and that of an actual circuit must be accepted. Nevertheless, the response computed with Ansoft Designer ${ }^{\circledR}$ in Fig. 2 shows very good agreement with that of Fig. 1. The simulated passband insertion loss is about $1.2 \mathrm{~dB}$, and the passband return loss is better than $10 \mathrm{~dB}$.

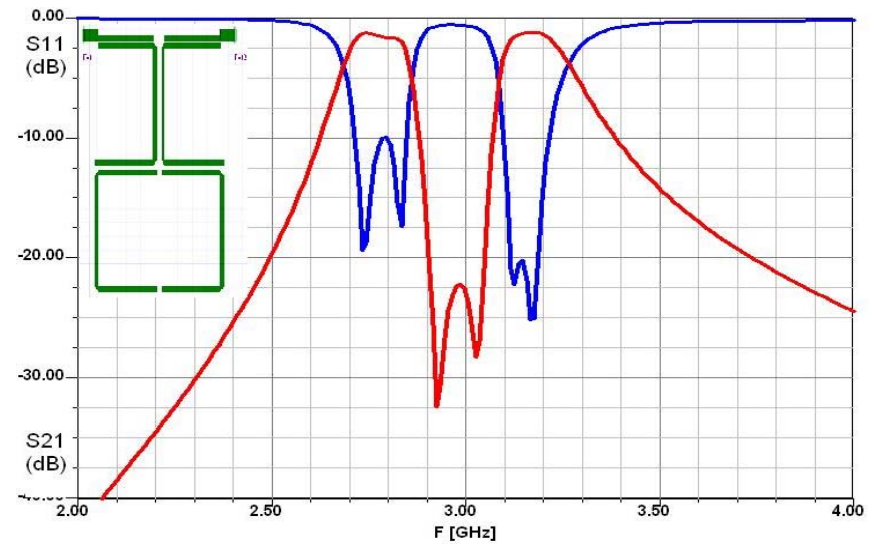

Fig. 2. Response of dual-band filter using hairpin resonators on RT6006 substrate.

\section{RESULTS}

As an extension of the previous example, Fig. 3 shows a triple-band filter using the same technology. The center frequencies of the three pass-bands are $2.65 \mathrm{GHz}, 3 \mathrm{GHz}$ and $3.35 \mathrm{GHz}$, and more than $50 \mathrm{MHz}$ bandwidth is obtained in each of the bands. Four transmission zeros are created by cross couplings between hairpin resonators 1-6 (magnetic transmission-line coupling) and 2-5 (electric open-end coupling). Two transmission zeros each are placed between adjacent pass-bands. Note that it is almost impossible to design such filters with other design theories.

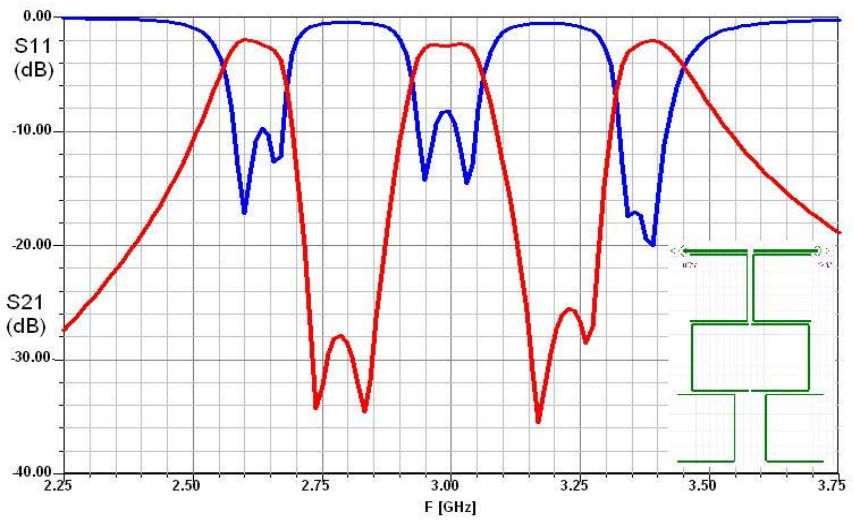

Fig. 3. Response of a triple-band filter using six hairpin resonators on RT6006 substrate and 1-6 / 2-5 cross coupling.

Out-of-band rejection can be controlled by placing transmission zeros not only between passbands but also to the left and/or right of all passbands. This is demonstrated in Fig. 4 using quasi-dual-mode resonators. The resulting response of the coupling matrix (Fig. 4a) shows four reflection and four transmission zeros which are obtained using source/load coupling. The coupling matrix for this type of filter is found as

$$
M=\left[\begin{array}{cccccc}
0 & 0.77 & 0 & 0 & 0 & 0.073 \\
0.77 & 0 & 0.99 & 0 & -0.72 & 0 \\
0 & 0.99 & 0 & 0 & 0 & 0 \\
0 & 0 & 0 & 0 & 0.99 & 0 \\
0 & -0.72 & 0 & 0.99 & 0 & 0.77 \\
0.073 & 0 & 0 & 0 & 0.77 & 0
\end{array}\right]
$$

with a center frequency at $3 \mathrm{GHz}$ and four percent bandwidth in each passband. The results simulated with Ansoft Designer $^{\circledR}$ are illustrated in Fig. 4b. The insertion loss within the passbands is less than $2.5 \mathrm{~dB}$, and the return loss is better than $15 \mathrm{~dB}$. In addition and as an improvement over the coupling matrix response, the quasi-dual-mode structure achieves $30 \mathrm{~dB}$ attenuation between the two passbands and in the stopbands to the left and right.

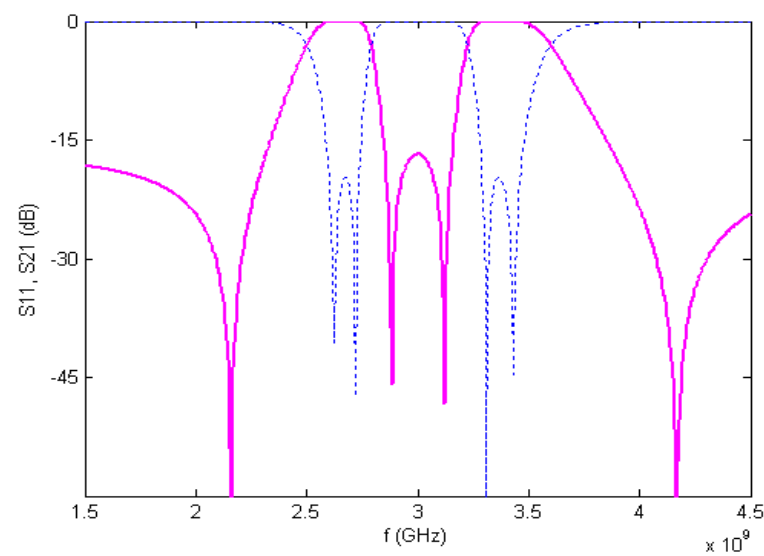

(a)

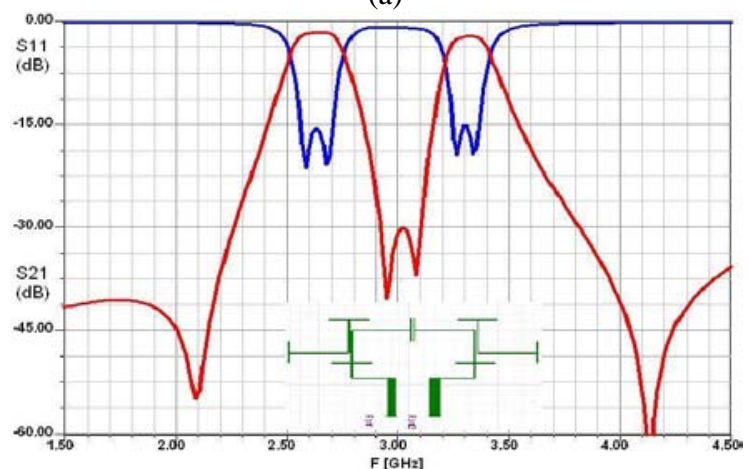

(b)

Fig. 4. Responses of four-pole dual-band filter using quasi-dualmode resonators: (a) coupling matrix, (b) Ansoft Designer.

In order to improve selectivity around each passband, a sixpole cross-coupled dual-band filter with hairpin resonators is designed with a center frequency at $3 \mathrm{GHz}$, five percent bandwidth in each of the passbands and transmission zeros at 2.4, 2.9, 3.1 and $3.7 \mathrm{GHz}$. The substrate is RT6006 as in previous examples. After applying the theory outlined in Section II, the following coupling matrix is obtained: 


$\mathrm{M}=\left[\begin{array}{cccccccc}0 & 0.9055 & 0 & 0 & 0 & 0 & 0 & 0 \\ 0.9055 & 0 & 0.7992 & 0.5812 & 0 & 0 & -0.1777 & 0 \\ 0 & 0.7992 & 0 & 0 & 0 & 0.0065 & 0 & 0 \\ 0 & 0.5812 & 0 & 0 & 0.9924 & 0 & 0 & 0 \\ 0 & 0 & 0 & 0.9924 & 0 & 0 & 0.5812 & 0 \\ 0 & 0 & 0.0065 & 0 & 0 & 0 & 0.7992 & 0 \\ 0 & -0.1777 & 0 & 0 & 0.5812 & 0.7992 & 0 & 0.9055 \\ 0 & 0 & 0 & 0 & 0 & 0 & 0.9055 & 0\end{array}\right]$

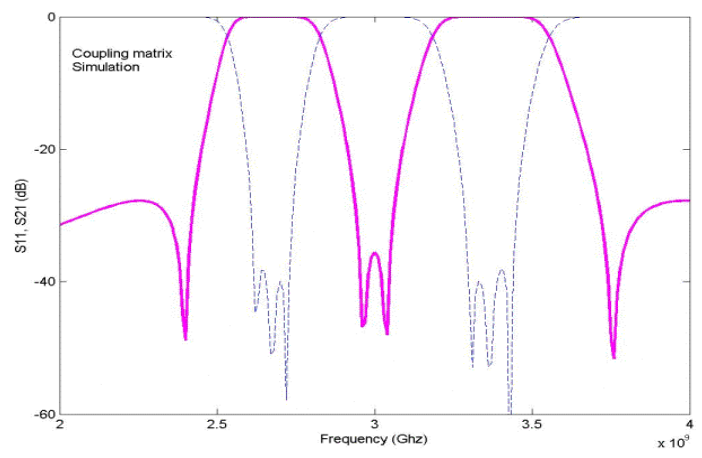

(a)

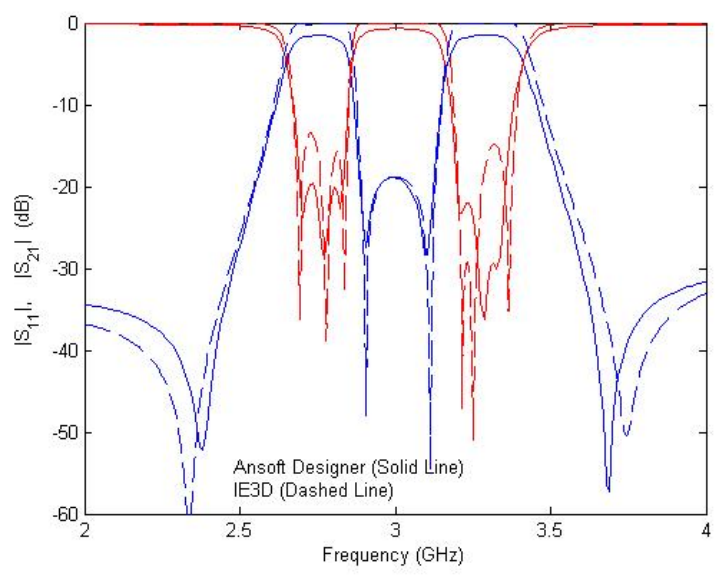

(b)

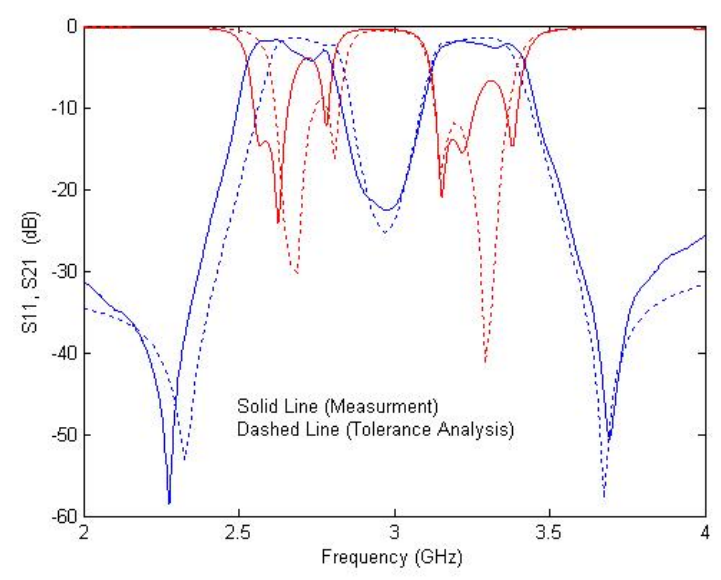

(c)

Fig. 5. Responses of six-pole dual-band filter using hairpin resonators: (a) coupling matrix, (b) IE3D (lossless) and Ansoft Designer (including losses), (c) measurements and tolerance analysis.
Figs. 5a and $5 \mathrm{~b}$ show the simulated responses from the coupling matrix and two EM-based software packages, respectively. Good agreement is observed. Slight differences between Ansoft Designer ${ }^{\circledR}$ and IE3D $^{\circledR}$ results have been observed previously [12] and are attributed to the different approaches of the method-of-moments implementation in both packages. The measurements in Fig. 5c confirm the design in principle but fail to reproduce the two transmission zeros between the passbands. This is attributed to the manufacturing tolerances, which were originally specified to be within $10 \mu \mathrm{m}$ but turned out to be $25 \mu \mathrm{m}$ and even slightly above. Therefore, a tolerance analysis was performed which is also shown in Fig. 5c. This analysis confirms the disappearance of the two transmission zeros, thus validating the design in principle. Of course, the cross couplings that create transmission zeros between passbands are sensitive to fabrication tolerances which are recommended to be in the order of $10 \mu \mathrm{m}$ for similar designs. Fig. 6 shows a photograph of the prototype on RT6006 substrate. The six individual hairpin resonators are clearly visible.

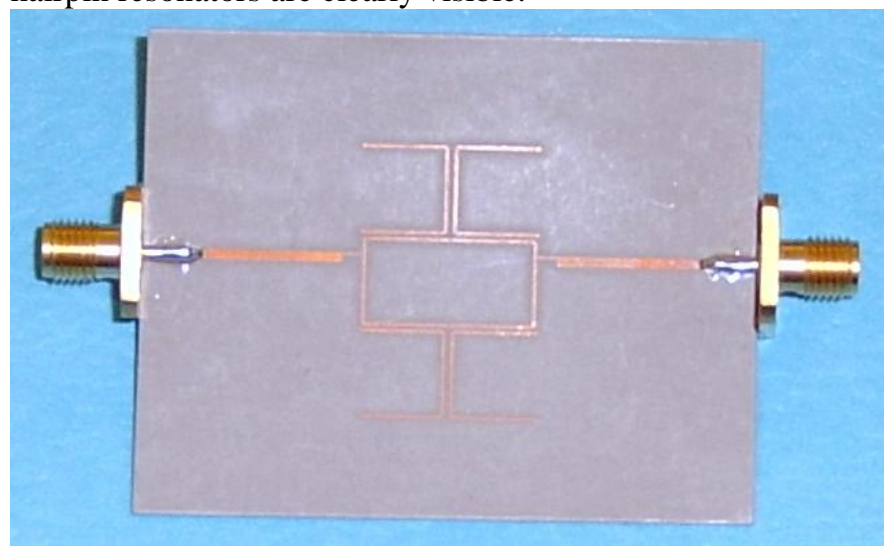

Fig. 6. Photograph of the prototype six-pole dual-mode filter using hairpin resonators.

The last example is a six-pole dual-band cross-coupled filter using open-loop resonators. The topology of the coupling matrix equals that of (3) with only slightly changes in the matrix elements. A photograph of his dual-band filter prototype is depicted in Fig. 7a. It is noted that the design is more compact than that of the previous example (Fig. 6). Fig. $7 \mathrm{~b}$ demonstrates good agreement between the simulation results of Ansoft Designer ${ }^{\circledR}$ and IE3D ${ }^{\circledR}$. Similar to the results of Fig. 5, the measurements presented in Fig. 7c fail to reproduce the two transmission zeros between the passbands. However, reasonable agreement with measurements is again observed once the effects of fabrication tolerances, which are shown in Fig. 7c for comparison, are taken into account.

\section{CONCLUSIONS}

The new coupling-matrix approach for uni-planar dual-band and triple-band filters presents a viable alternative to current 


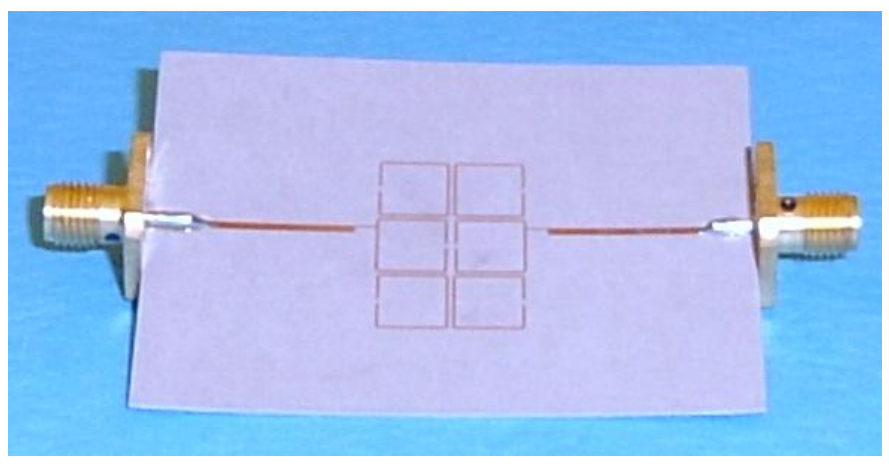

(a)

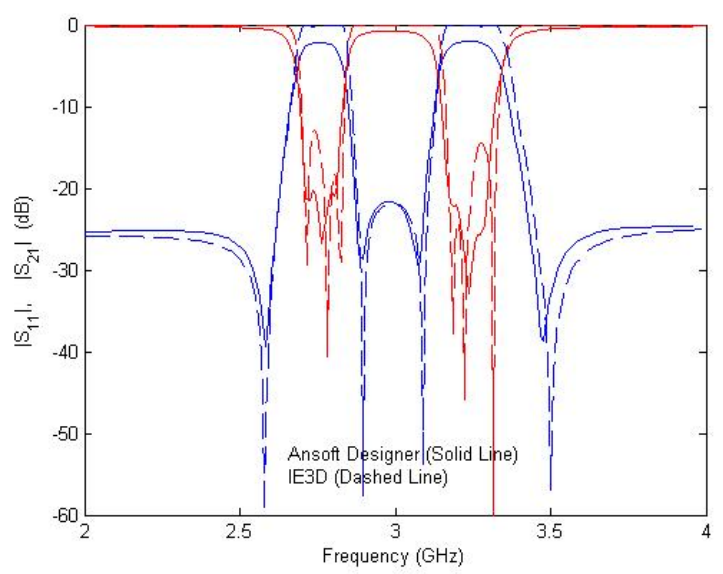

(b)

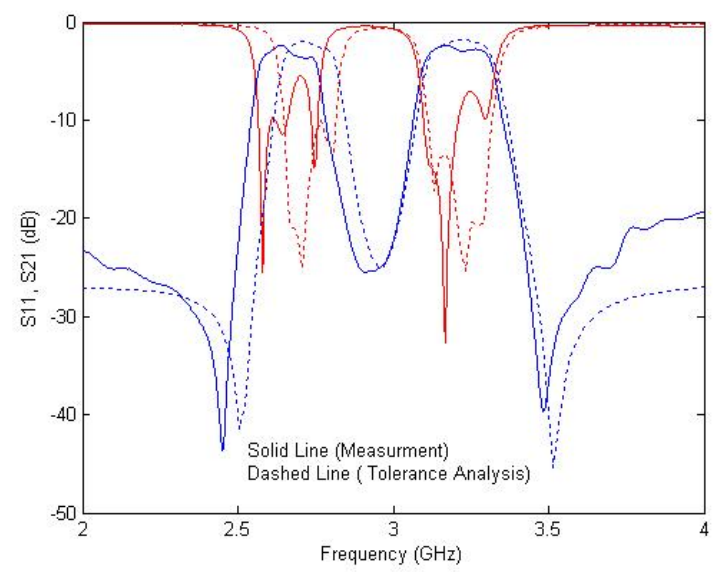

(c)

Fig. 7. Six-pole dual-band filter using open-loop resonators: (a) photograph, (b) IE3D (lossless) and Ansoft Designer (including losses) results, (c) measurements and tolerance analysis. multiband design techniques. Of special advantage are the specification of the coupling topology and the enforcement of coupling coefficients during the design stages. The results demonstrate the feasibility of the technique for hairpin, openloop and quasi-dual-mode resonator configurations. The designs presented are verified by commercially available field solvers and, in principle, by measurements.

\section{REFERENCES}

[1] H. Miyake, S. Kitazawa, T. Ishizaki, T. Yamanda, Y. Nagatomi, "A miniaturized monolithic dual-band filter using ceramic lamination technique for dual-mode portable telephones", IEEE MTT-S Int. Microwave Symp. Dig., pp. 789-792, June 1997.

[2] C. Quendo, E. Ruis, C. Person, "An original topology of dualband filter with transmission zeros", IEEE MTT-S Int. Microwave Symp. Dig., pp. 1093-1096, June 2003.

[3] S.F. Chang, Y.H. Jeng, J.L. Chen, "Dual-band step-impedance bandpass filter for multimode wireless LANs", IEE Electronics Lett., Vol. 40, pp. 38-39, Jan. 2004.

[4] C.C. Chen, "Dual-band bandpass filter using coupled resonator pairs", IEEE Microwave Wireless Comp. Lett., Vol.15, pp. 259261, Apr. 2005.

[5] A.E. Atia and A.E. Williams, "New type of waveguide band pass filters for satellite transponders", COMSAT Tech. Rev., Vol. 1, no. 1, pp. 21-43, 1971.

[6] S. Amari, U. Rosenberg and J. Bornemann, "Adaptive synthesis and design of resonator filters with source/load-multiresonator coupling", IEEE Trans. Microwave Theory Tech., Vol. 50, pp. 1969-1978, Aug. 2002

[7] E.G. Cristal, S. Frankel, "Hair-pin line and hybrid hair-pin line/half-wave parallel-coupled-line filters", IEEE Trans. Microwave Theory Tech., Vol. 20, pp. 719-728, Nov. 1972.

[8] J.S. Hong, M.J. Lancaster, "Aperture-coupled microstrip openloop resonators and their applications to the design of novel microstrip band-pass filters", IEEE Trans. Microwave Theory Tech., Vol. 47, pp. 1848-1855, Sept. 1999.

[9] R.R. Mansour, S. Ye, S.F. Peik, V. Dokas, B. Fitzpatrick, "Quasi-dual-mode resonators", IEEE Trans. Microwave Theory Tech., Vol. 48, pp. 2476-2480, Dec. 2000.

[10] S. Amari, "Synthesis of cross-coupled resonator filters using an analytical gradient-based optimization technique", IEEE Trans. Microwave Theory Tech., Vol. 48, pp. 1559-1564, Sep. 2000.

[11] J.S. Hong, M.J. Lancaster, "Coupling of microstrip square openloop resonators for cross-coupled planar microwave filters", IEEE Trans. Microwave Theory Tech., Vol.44, pp. 2099-2109, Nov. 1996.

[12] K. Rambabu and J. Bornemann, "Simplified analysis technique for the initial design of a class of LTCC filters", IEEE Trans. Microwave Theory Tech., Vol. 53, pp. 1787-1791, May 2005. 